Alibert, M. 37

Aquilina, C. 37

Boisnic, S. 43

Bonafé, J.L. 25

Borrel, M.-T. 53

Branchet-Gumila, M.-C. 43

Briant, A. 37

Charvéron, M. 25

Creidi, P. 49

Dahan, S. 37

Denis, P. 37

Didierjean, L. 13, 19

Diridollou, S. 37

Dupuy, P. 37, 53, 57
Fluhr, J.W. 57

Fort-Lacoste, L. 33

Gall, Y. 25, 53

Gehring, W. 57

Germanier, L. 29

Gloor, M. 57

Humbert, Ph. 49

Lachgar, S. 25

Launais, B. 37

Lauze, C. 53, 57

Le Charpentier, Y. 43

Navarro, R. 33

Ochando, N. 53
Pechère, J.-C. 29

Pechère, M. 29

Sachsenberg-Studer, E.M. 61

Saurat, J.-H. 1, 13, 19, 29

Segard, C. 43

Siegenthaler, G. 29

Sorg, O. 1, 13, 19

Tisne-Versailles, J. 33

Tran, C. 19

Turlier, V. 37

Vahlquist, A. 3

Verscheure, Y. 33

Vienne, M.-P. 37, 53, 57

\title{
Subject Index Vol. 199, S1, 1999
}

Acne 33

Biological activities 19

Citral 19

Collagen 43

Echography 37

Elastic fibers 43

Elasticity 37

Epidermis 13

Ex vivo model 43

Irritation 57

Local tolerance 57

Metabolism 13

Mouse tail model 19

Myeloperoxidase 19
Natural retinoids 3 Normal human skin 43

Organ culture 43

Patch-testing 57 Photoaging 37, 43, 49

Propionibacterium acnes 29

Retinaldehyde 3, 13, 25,33, $37,43,49,53,57,61$

Retinaldehyde, 9-cis 19

- topical 19

Retinoic acid 57, 61

Retinoid 49

Retinoid irritation 19

Retinoids 13

Retinol 57,61

Rhino mouse 33

Rosacea 53
Safety 61

Skin thickness 37

Stiffness 37

Tolerance 61

Topical preparation 33

Topical retinaldehyde 29

- retinoids 53

Ultraviolet light 43

Vascular endothelial growth factor 25

Vitamin A 3

\begin{tabular}{ll}
\hline KARGER & @ 1999 S. Karger AG, Basel \\
Fax +41 61 306 12 34 & 1018-8665/99/1997-0064\$17.50/0 \\
$\begin{array}{l}\text { E-Mail karger@karger.ch } \\
\text { www.karger.com }\end{array}$ & Accessible online at: \\
http://BioMedNet.com/karger
\end{tabular}

